\title{
НЕСТАЦИОНАРНАЯ ЗАДАЧА ТЕПЛООБМЕНА ДЛЯ УРАВНЕНИЯ ПАРАБОЛИЧЕСКОГО ТИПА В НЕЦИЛИНДРИЧЕСКОЙ ОБЛАСТИ
}

\author{
@ Р.Г. Зайнуллин \\ zaynulin_r.g@mail.ru
}

УДК 517.95

DOI: $10.33184 /$ mnkuomsh2t-2021-10-06.73.

Излагается применение метода разложения по собственным функциям самосопряженного дифференциального оператора к решению одной нестационарной задачи теплообмена с фазовым переходом в неавтомодельной постановке при специальных начальных условиях на примере процесса промерзания некоторой сплошной среды. В ходе решения задачи устанавливается параболический закон движения границы раздела двух фаз. Задачи подобного типа возникают при математическом моделировании процессов теплообмена в строительстве, особенно в районах вечной мерзлоты, в нефтегазодобыче при бурении и эксплуатации скважин, в металлургии и т.д.

Ключевые слова: Фазовый переход, свободные границы, движущиеся границы, задача Стефана, конечные интегральные преобразования, вырожденные гипергеометрические функции, возмущенный дифференциальный оператор.

\section{NON-STATIONARY HEAT TRANSFER PROBLEM FOR A PARABOLIC TYPE EQUATION IN A NON-CYLINDRICAL DOMAIN}

The application of the eigenfunction decomposition method of a selfadjoint differential operator to the solution of a non-stationary heat transfer problem with a phase transition in a non-automatic formulation under special initial conditions is presented on the example of the freezing process of a continuous medium. In the course of solving the problem, a parabolic law of motion of the interface of two phases is established. Problems of this type arise in the mathematical modeling of heat exchange processes in construction, especially in permafrost areas, in oil and gas production during drilling and operation of wells, in metallurgy, etc.

Keywords: Phase transition, free boundaries, moving boundaries, Stefan problem, finite integral transformations, degenerate hypergeometric functions, perturbed differential operator.

Зайнуллин Рифат Гильметдинович, к.ф.-м.н., доцент, УГАТУ (Уфа, Россия); Rifat Zaynulin (Ufa State Aviation Technical University, Ufa, Russia) 
Рассмотрим задачу о динамике зон кристаллизации $(k=1)$ и охлаждения $(k=1)$ некоторой сплошной среды под действием точечного источника холода, когда граница фазового переход имеет сферическую форму и с течением времени продвигается внутрь среды. В начальный момент времени среда обладает постоянной температурой $t_{0}>0$ при $r \geq a \xi_{0}$. Точечный источник обладает температурой $t_{e}<0$. Математическую модель этого процесса для радиальной схемы можно представить в виде:

$$
\begin{gathered}
\frac{1}{a_{k}^{2}} \frac{\partial t_{k}(r, \tau)}{\partial \tau}=\frac{\partial^{2} t_{k}(r, \tau)}{\partial r^{2}}+\frac{2}{r} \frac{\partial t_{k}(r, \tau)}{\partial r} \\
r \in D_{k}(\tau): \quad D_{1}(\tau)=\left\{0<r<\xi_{1}(\tau)\right\}, \quad D_{2}(\tau)=\left\{\xi_{1}(\tau)<r<\xi_{2}(\tau)\right\} \\
\tau>0, \xi_{1}(+0)=\xi_{0}>0, \xi_{2}(\tau)=a \xi_{1}(\tau), a>1 \\
t_{1}(r, 0)=\left(1-\frac{r}{\xi_{0}}\right) t_{e} ; \quad t_{2}(r, 0)=\frac{r-\xi_{0}}{(a-1) \xi_{0}} t_{0} \\
t_{1}(0, \tau)=t_{e} ; \quad t_{k}\left(\xi_{1}(\tau), \tau\right)=0 ; \quad t_{2}\left(\xi_{2}(\tau), \tau\right)=t_{0} \\
\lambda_{1} \frac{\partial t_{1}\left(\xi_{1}(\tau), \tau\right)}{\partial r}-\lambda_{2} \frac{\partial t_{2}\left(\xi_{1}(\tau), \tau\right)}{\partial r}=\sigma \nu \frac{d \xi_{1}(\tau)}{d \tau}
\end{gathered}
$$

Здесь $a_{k}$ и $\lambda_{k}$ - коэффициенты температуропроводности и теплопроводности в $D_{k}(\tau), \sigma$ - скрытая теплота кристаллизации, а $\nu$ - плотность образующейся фазы, $a$ - безразмерный параметр теплового влияния [18]. Требуется найти функции $t_{k}(r, \tau)$ и $\xi_{1}(\tau)$, такие, что имеют место равенства (1)-(4).

Для решения задачи вводится подвижная граница; задача преобразуется к области с неподвижными границами с неоднородной правой частью и переменными коэффициентами в исходном уравнении, но с однородными краевыми условиями, и далее, для решения преобразованной задачи строится интегральное преобразование по пространственной координате с неизвестным ядром, нахождение которого связано с постановкой и решением соответствующей спектральной задачи через вырожденные гипергеометрические функции. Находятся собственные функции и собственные числа, а также формула для обращения для введенного интегрального преобразования, что позволяет выписать аналитическое решение исходной задачи и рассмотреть ряд частных случаев. 


\section{Литература}

1. Шафеев M.Н. Решение одной плоской задачи Стефана методом ВГГП // Инженерно-физический журнал, 1978, Т. 34, № 4, С. 713-722.

2. Шафеев М.Н. Решение одной нелинейной задачи методом ВГГП // Известия вузов. Математика, 1980, № 12(233), С. 73-75.

3. Хакимов Р.Х. Замораживание грунтов в строительных целях. М.: Госстройиздат, 1962, с.257.

4. Зайнуллин Р.Г. Об одном аналитическом подходе к решению одномерной задачи переноса тепла со свободными границами // Известия вузов. Математика, 2008, № 2, С. 24-31.

5. Зайнуллин Р.Г., Фазуллин З.Ю. Краевая задача для уравнения параболического типа в нецилиндрической области // Мат.заметки СВФУ, т.27, №2,апр.-июн., 2020, С.3-20. 